\title{
Study of Cellulose Nanocrystals and Zinc Nitrate Hexahydrate Addition in Chitosan Hydrogels
}

\author{
Sheila de Oliveira Ferreira ${ }^{a^{\star}} \oplus$, Thaís Larissa do Amaral Montanheiro ${ }^{b, a} \oplus$, \\ Larissa Stieven Montagna ${ }^{a}{ }^{\circledR}$, Lilia Müller Guerrinic, Ana Paula Lemes ${ }^{a}{ }^{\circledR}$ \\ ${ }^{a}$ Laboratório de Tecnologia de Polímeros e Biopolímeros, Instituto de Ciência e Tecnologia, \\ Universidade Federal de São Paulo, São José dos Campos, SP, Brasil \\ ${ }^{b}$ Divisão de Ciências Fundamentais, Instituto Tecnológico de Aeronáutica, São José dos Campos, SP, \\ Brasil \\ 'Laboratório de Processos e Caracterização de Polímeros, Instituto de Ciência e Tecnologia, \\ Universidade Federal de São Paulo, São José dos Campos, SP, Brasil
}

Received: December 04, 2018; Accepted: March 09, 2019

In this study, chitosan hydrogels were produced with $0,2,4$ and $6 \mathrm{wt} \%$ of cellulose nanocrystals $(\mathrm{CNC})$ using hexahydrate zinc nitrate $\left(\mathrm{Zn}\left(\mathrm{NO}_{3}\right)_{2} \cdot 6 \mathrm{H}_{2} \mathrm{O}\right)$ as a catalyst for chitosan crosslinking reaction. CNC's size was estimated by dynamic light scattering (DLS) and surface charge by zeta potential. Hydrogels were characterized by rotational rheometer, swelling test, uniaxial compression test, in vitro degradation, scanning electron microscopy (SEM) and microtomography. Results showed that zinc nitrate and $\mathrm{CNC}$ addition did not influence mechanical properties, degradation, and morphology of the hydrogels. However, zinc nitrate decreased $36.54 \%$ of the gel time and $41.37 \%$ of the swelling degree, and increased the crosslinking degree of the chitosan hydrogels, proving not only its catalytic effect but also its participation in the crosslinking reaction. Porosity was slightly reduced after addition of zinc nitrate and incorporation of $\mathrm{CNC}$. In the mechanism of crosslinking reaction, a competition between $\mathrm{CNC}$ and zinc nitrate was observed.

Keywords: chitosan hydrogel, crosslinking, glutaraldehyde, zinc nitrate, cellulose nanocrystals.

\section{Introduction}

Hydrogels designed for medical applications are inherently biocompatible due to the hydrophilicity and anatomical similarity to humans, so, they can be used as a biomaterial to simulate a bone, cartilage, and others. They are three-dimensional polymer networks capable of swelling in an aqueous environment without dissolving, and they are formed by crosslinking between polymer chains, using a crosslinking agent ${ }^{1,2}$.

Chitosan is a natural polymer of great interest in the production of hydrogels for medicine due to its biocompatibility, biodegradability and bacteriostatic activity. It can be used in polymeric scaffolds for tissue engineering, wound healing, microcapsules for drug controlled release and many others medical applications ${ }^{3-5}$. This polymer is also sustainable, cheap and easy to find, once it is obtained from the chitin, the second most abundant natural polymer in the biosphere ${ }^{3}$.

Glutaraldehyde is the primary agent used in chitosan hydrogels crosslinking ${ }^{6,7}$. This reaction is more efficient when using catalysts such as zinc nitrate $\left(\mathrm{Zn}\left(\mathrm{NO}_{3}\right)_{2}\right)^{8,9}$. Zinc nitrate is a material of low toxicity and often used in the synthesis of catalysts, for example, in carbon nanotubes and biodiesel ${ }^{10,11}$.

Chitosan has low mechanical strength, then, many studies have added nanoparticles to their matrices to improve properties. An example of nanoparticle used is the cellulose nanocrystal $(\mathrm{CNC})^{12,13}$

Cellulose is the renewable organic material most abundant in the biosphere. It is a fibrous substance that has crystalline and amorphous regions. When the crystalline regions are isolated by enzymatic or acid hydrolysis, CNCs are obtained ${ }^{14-16}$

$\mathrm{CNC}$ possess many desirable properties, such as large surface area, excellent colloidal stability, high tensile strength and stiffness, and hydroxyl groups that have potential for reactions ${ }^{17}$. They are needle-shaped nanostructures used in composites as reinforcement to improve essential properties in materials such as thermal stability, water and mechanical resistance ${ }^{18-22}$. They can be used for drug delivery, protein and enzyme immobilization, support cell cultures, biosensing and bioethanol production ${ }^{17,23-25}$.

When used as a crosslinking agent, glutaraldehyde may react with amine groups $\left(-\mathrm{NH}_{2}\right)$ present in chitosan and with the hydroxyls groups (-OH) present in the $\mathrm{CNC}$, making possible different reactions like $\mathrm{CNC}-\mathrm{CNC}$ molecules, $\mathrm{CNC}$ chitosan molecules and between the chitosan molecules ${ }^{26}$.

$\mathrm{CNC}$ addition into chitosan matrices can improve its properties, and the use of $\mathrm{Zn}\left(\mathrm{NO}_{3}\right)_{2}$ as a catalyst can increase the crosslinking efficiency ${ }^{9,11,19}$. To our knowledge, there is no report in the literature about chitosan/cellulose nanocrystals hydrogels nanocomposites using glutaraldehyde as the crosslinking agent and zinc nitrate as the catalyst, 
what makes, therefore, this work innovative and important regarding the hydrogels research.

\section{EXPERIMENTAL}

\subsection{Materials}

Chitosan with minimum deacetylation degree of $95 \%$ was supplied by Primex(r), glutaraldehyde from SigmaAldrich and zinc nitrate hexahydrate $\left(\mathrm{Zn}\left(\mathrm{NO}_{3}\right)_{2} \cdot 6 \mathrm{H}_{2} \mathrm{O}\right)$ from Synth(r). CNC, produced by acid hydrolysis and dried by spray dryer, were supplied by CelluForce.

\subsection{Characterization of $\mathrm{CNC}$}

\subsubsection{Dynamic Light Scattering (DLS) and Zeta Potential}

The average particle diameter and zeta potential values were obtained on a Beckman Coulter Delsa Nano equipment. $\mathrm{CNC}$ were suspended in deionized water, and the measurements were performed at $25^{\circ} \mathrm{C}$ at an angle of $15^{\circ}$. For the zeta potential calculation from mobility values, the Smoluchowski equation was used, with refractive index, dielectric constant and water viscosity values at $25^{\circ} \mathrm{C}$.

\subsection{Production of Chitosan/CNC Hydrogels}

A solution of chitosan $1 \%(\mathrm{w} / \mathrm{v})$ with acetic acid $2 \%(\mathrm{v} / \mathrm{v})$, hexahydrate zinc nitrate $\left(\mathrm{Zn}\left(\mathrm{NO}_{3}\right)_{2} \cdot 6 \mathrm{H}_{2} \mathrm{O}\right)$ and different volumes of CNC suspension was prepared to obtain nanocomposites with the desired CNC concentration (2, 4 and $6 \mathrm{wt} \%$ ). This solution was kept in magnetic stirring during $1 \mathrm{~h}$, and then it was sonicated for $5 \mathrm{~min}$ in an ultrasonic bath (Logen Scientific LS UC2-120-3.0). After, the glutaraldehyde was added with vigorous stirring for $1 \mathrm{~min}$. The resulting solution was poured into tubes internally covered with aluminum foil and kept for $24 \mathrm{~h}$. Then, the hydrogels were manually removed and washed three times with deionized water.

The molar ratio of glutaraldehyde:amine groups in chitosan was $0.7: 1(\mathrm{~mol} / \mathrm{mol})$ and between $\mathrm{Zn}\left(\mathrm{NO}_{3}\right)_{2} \cdot 6 \mathrm{H}_{2} \mathrm{O}$ :glutaraldehyde was $0.05: 1(\mathrm{~mol} / \mathrm{mol})$. The hydrogels were produced according to Table 1 .

\subsection{Characterization of Chitosan/CNC Hydrogels}

\subsubsection{Rheological Measurements}

The crosslinking hydrogels were studied by dynamic viscoelastic behavior as a function of time. The viscoelastic

Table 1. Hydrogels description

\begin{tabular}{cc}
\hline Hydrogel & Description \\
\hline C & Without zinc nitrate and without CNC \\
C/N & With zinc nitrate and without CNC \\
C/N/CNC 2 & With zinc nitrate and $2 \% \mathrm{CNC}$ \\
C/N/CNC 4 & With zinc nitrate and $4 \% \mathrm{CNC}$ \\
C/N/CNC 6 & With zinc nitrate and $6 \% \mathrm{CNC}$ \\
\hline
\end{tabular}

behavior was analyzed by dynamic stress sweep test at constant angular frequency ( $1 \mathrm{hz}$ ) and temperature in the range from 0 to $70 \mathrm{~Pa}$ in the sample of quitosan without glutaraldehyde, zinc nitrate and cellulose nanocrystals.

The gel time was determined by dynamic time sweep tests at $0.1 \mathrm{~Pa}$ and $25 \pm 0.5^{\circ} \mathrm{C}$ using a rotational rheometer (Haake - Reostress 6000) with parallel plates geometry.

\subsubsection{X-Ray Microtomography}

3D images and porosity values were obtained by X-ray microtomography (SkyScan 1272 Bruker microCT, Kontich, Belgium) with pixel resolution of $7.4 \mu \mathrm{m}$. The X-ray source was defined $20 \mathrm{kV}$ of energy and $175 \mu \mathrm{A}$ of current. Images of the cross sections of the hydrogels were obtained with a XIMEA xiRAY16 (2419 dpi) camera. Porosity values were obtained using a CTAn software (Bruker). Round cuttings of $500 \mu \mathrm{m}$ (diameter) and with fixed height were made in different points of the hydrogels. Five measurements were performed, and the results are given as the average value. The samples were previously lyophilized to perform the analysis.

\subsubsection{Scanning Electron Microscopy (SEM)}

The hydrogels surface fracture morphology was studied in a FEI - Quanta Scanning Electron microscope, operating at $15 \mathrm{KeV}$, with secondary electron detectors. The samples were frozen at $-80^{\circ} \mathrm{C}$, dried for 24 hours in a Labconco FreeZone 2.5 Plus lyophilizer and, then, fractured in liquid nitrogen.

\subsubsection{Water Absorption Capacity Test (Swelling Test)}

The hydrogels were immersed in distilled water and placed in an incubator at $37^{\circ} \mathrm{C}$ and $120 \mathrm{rpm}$ of agitation. The samples were removed and weighed every 15 minutes until the sample had a constant mass. The assay was performed in triplicate, and the swelling degree (SD) was calculated using the Equation 1.

$$
S D(\%)=\frac{M_{t}-M_{o}}{M_{o}} \times 100
$$

Where $\mathrm{M}_{\mathrm{t}}$ is the swollen hydrogel mass at " $\mathrm{t}$ " time and $\mathrm{M}_{0}$ is the dry hydrogel mass.

\subsubsection{Uniaxial Compression Test}

The uniaxial compression properties of the produced hydrogels were analyzed in a CT3 Texture Analyzer. The cylindrical samples had $10 \mathrm{~mm}$ of diameter and height. They were submitted to uniaxial compression with speed of $0,2 \mathrm{~mm} \mathrm{~s}^{-1}$ until fracture. The assay was performed at room temperature.

\subsubsection{In Vitro Degradation}

To evaluate in vitro degradation behavior, hydrogels were placed in saline solution ( $\mathrm{pH} \mathrm{6)}$ for 70 days. In the first 28 days, the hydrogels were removed from the solution every 7 days, washed with distilled water, dried with absorbent 
paper and weighed; but from $29^{\text {th }}$ to $70^{\text {th }}$ day, this procedure occurred every 14 days. The saline solution was changed at each weighing. The test was performed on 5 samples of each hydrogel. Weight loss evaluated the degradationas a function of the time. In Equation 2, M is the percentage of remaining mass in relation to the initial mass, $\mathrm{M}_{0}$ is the initial hydrogel mass and $\mathrm{M}_{\mathrm{f}}$ is the hydrogel mass after degradation.

$$
M(\%)=\frac{M_{f}}{M_{o}} \times 100
$$

\section{Results and Discussions}

\subsection{Cellulose Nanocrystals Characterization}

\subsubsection{Dynamic Light Scattering (DLS) and Zeta Potential}

The average $\mathrm{CNC}$ particle size in water suspension estimated by dynamic light scattering was $118 \mathrm{~nm}$ with a polydispersity of 0.317 . The zeta potential in deionized water was - $45 \mathrm{mV}$. Then, this might be considered a stable CNC suspension, because the modulus of the obtained value is greater than $25 \mathrm{mV}^{27}$. This value of zeta potential is suitable for the production of aqueous chitosan solution, once it will result in a stable colloidal suspension, resulting in a good dispersion of CNC into de hydrogels.

Although DLS technique assumes that each particle has spherical shape to calculate the hydrodynamic diameter, results can be used to prove the nanometric size and to compare particle size from others studies. Montanheiro et al. reported average diameter for $\mathrm{CNC}$ between 42 and $130 \mathrm{~nm}^{28}$, which is in accordance with the results obtained.

\subsection{Chitosan/Cellulose Nanocrystals Hydrogels Characterization}

\subsubsection{Rheological Measurements}

The rheological properties of the hydrogels were studied by dynamic viscoelastic behavior as a function of time.

Figure 1 shows the viscoelastic test of the chitosan without zinc nitrate, $\mathrm{CNC}$ and glutaraldehyde (uncrosslinked hydrogel), that is the solution of chitosan used in hydrogels production. Viscoelastic behavior was found at $0.1 \mathrm{~Pa}$. Thus, this stress was used to analyze gel point of the hydrogels.

Gel point is defined as the beginning of cross-linking during a gelation reaction, in which elastic and viscous modulus ( $G$ 'and $\left.G^{\prime \prime}\right)$ intersect, and, from that instant on, $\mathrm{G}^{\prime}$ becomes larger than $\mathrm{G}^{\prime \prime 2}$. Figure 2 (a-e) shows dynamic time sweep tests with $\mathrm{G}$ 'and $\mathrm{G}^{\prime \prime}$ of the hydrogels $\mathrm{C}, \mathrm{CN}$, $\mathrm{C} / \mathrm{N} / \mathrm{CNC} 2, \mathrm{C} / \mathrm{N} / \mathrm{CNC} 4$ and $\mathrm{C} / \mathrm{N} / \mathrm{CNC} 6$. Gel time (t gel) and critical shear modulus $(\mathrm{Gc})$ values of this samples are in Table 2.

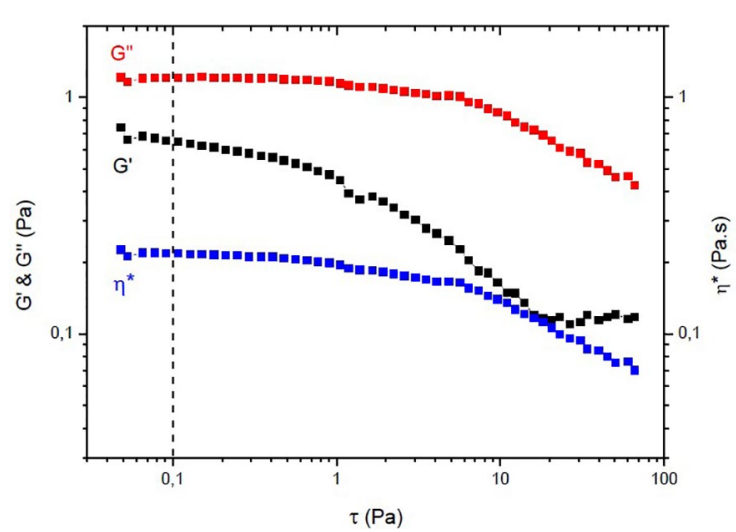

Figure 1. Dynamic stress sweep test of the uncrosslinked chitosan hydrogel.

According to Figure 2 and Table 2, it can be observed that the gel time, comparing sample $\mathrm{C}$ to $\mathrm{CN}$, decreased $36.54 \%$, confirming the catalytic effect of the zinc nitrate ${ }^{8,9}$. However, when $\mathrm{CNC}$ was added, for all concentrations, the gel time increased compared to the $\mathrm{C} / \mathrm{N}$ sample. Glutaraldehyde may react with amine groups $\left(-\mathrm{NH}_{2}\right)$ present in chitosan and with the hydroxyls groups $(-\mathrm{OH})$ present in the $\mathrm{CNC}$, making possible different reactions like $\mathrm{CNC}-\mathrm{CNC}$ molecules, $\mathrm{CNC}$ chitosan molecules and between the chitosan molecules. This behavior can generate competition in the mechanism of the crosslinking reaction, changing its kinetics and its efficiency ${ }^{26}$.

The graphs of the complex viscosity $\left(\eta^{*}\right)$ as a function of time ( $t$ ) of the crosslinked chitosan hydrogels are shown in Figure 3. It is observed that the zinc nitrate addition also increased the viscosity of hydrogel (samples $\mathrm{C}$ and NC). The increasing of viscosity can be related to an increase in the crosslinking degree of the chitosan hydrogels. It was proved that, zinc nitrate not only act decreasing the crosslink time but also contributed increasing the crosslinking degree of the chitosan hydrogel. However, $\mathrm{CNC}$ addition decreased the hydrogels viscosity and also the crosslinking degree. This behavior can also be explained by competition in the mechanism of the crosslinking reaction, previously mentioned ${ }^{26}$. Therefore, increasing $\mathrm{CNC}$ concentration in $\mathrm{C} / \mathrm{N}$ hydrogel causes, at first, a decrease in viscosity of hydrogel, as verified for $\mathrm{C} / \mathrm{N} / \mathrm{CNC} 2$ and $\mathrm{C} / \mathrm{N} / \mathrm{CNC} 4$ hydrogels (Figure 3).

Opposite behavior was found by Zhou et al..$^{30-31}$ with polyacrylamide-CNC hydrogels. The $\mathrm{CNC}$ accelerated the formation of hydrogels and increased the effective crosslink density of hydrogels. Thus, the authors found that $\mathrm{CNC}$ were not only a reinforcing agent for hydrogel, but also acted as a multifunctional cross-linker for gelation.

Addition of fillers in polymer solutions hampers the polymer chairs flow and increase viscosity of the mixture. This way, although $\mathrm{CNC}$ filler causes a decrease of viscosity due the decrease of crosslinking degree, higher $\mathrm{CNC}$ concentrations 

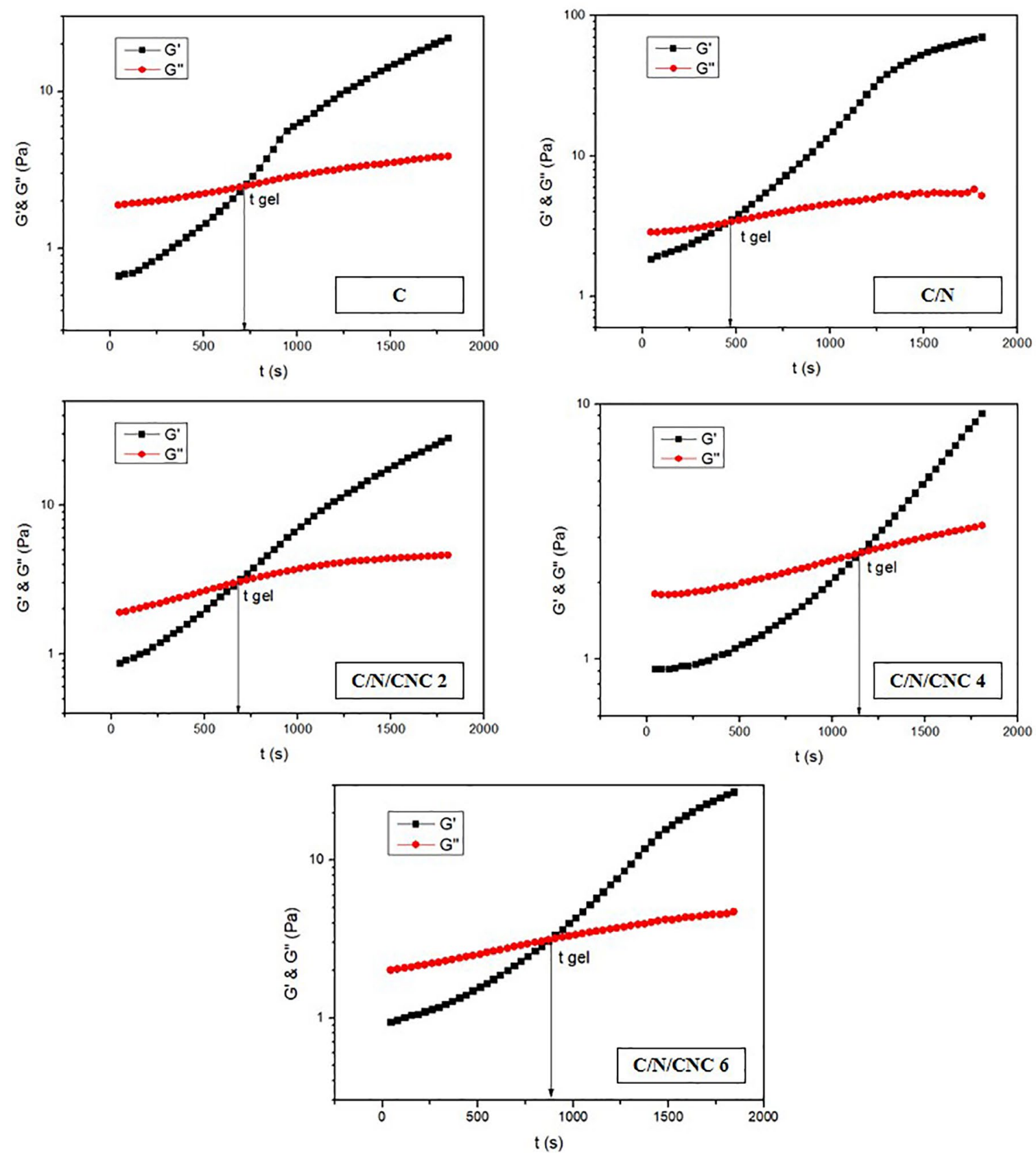

Figure 2. Time dependence of $\mathrm{G}^{\prime}$ and $\mathrm{G}$ " for the $\mathrm{C}, \mathrm{C} / \mathrm{N}, \mathrm{C} / \mathrm{N} / \mathrm{CNC} 2, \mathrm{C} / \mathrm{N} / \mathrm{CNC} 4$ and $\mathrm{C} / \mathrm{N} / \mathrm{CNC} 6$ hydrogels.

Table 2. Gel time (t gel) and critical shear modulus $(\mathrm{Gc})$ values of the hydrogels.

\begin{tabular}{ccc}
\hline Hydrogel & t gel (s) & Gc (Pa) \\
\hline $\mathrm{C}$ & 717 & 2.51 \\
$\mathrm{C} / \mathrm{N}$ & 455 & 3.40 \\
$\mathrm{C} / \mathrm{N} / \mathrm{CNC} \mathrm{2}$ & 676 & 3.04 \\
$\mathrm{C} / \mathrm{N} / \mathrm{CNC} 4$ & 1152 & 2.63 \\
$\mathrm{C} / \mathrm{N} / \mathrm{CNC} 6$ & 905 & 3.36 \\
\hline
\end{tabular}

promote, simultaneously, an increase in the viscosity of the mixture. Therefore, $\mathrm{C} / \mathrm{N} / \mathrm{CNC} 6$ hydrogel showed higher viscosity than $\mathrm{C} / \mathrm{N} / \mathrm{CNC} 4$ hydrogels.

\subsubsection{X-Ray Microtomography}

Figure 4 shows 3D images of the hydrogels obtained by $\mathrm{X}$-ray microtomography. It can be observed, for all samples, highly porous morphology, without density variations, since 
X-rays have distinct behavior when passing through solids with different densities. So, in the case of density variations, it would be possible to observe color changes in the scaffolds. It may be concluded then, that CNCs were satisfactorily dispersed among the samples ${ }^{32}$.

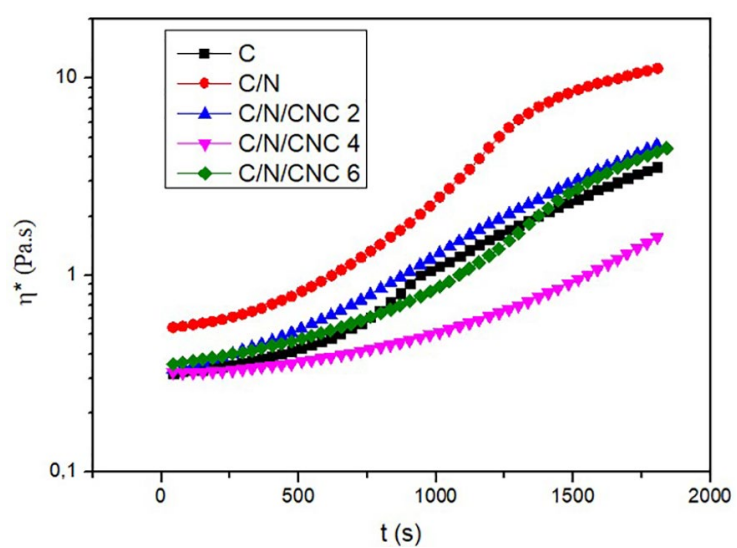

Figure 3. Graph of the complex viscosity $\left(\eta^{*}\right)$ as a function of time (t) of the hydrogels.
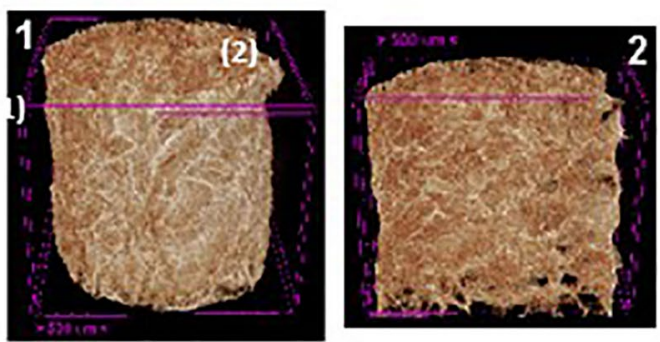

(C)
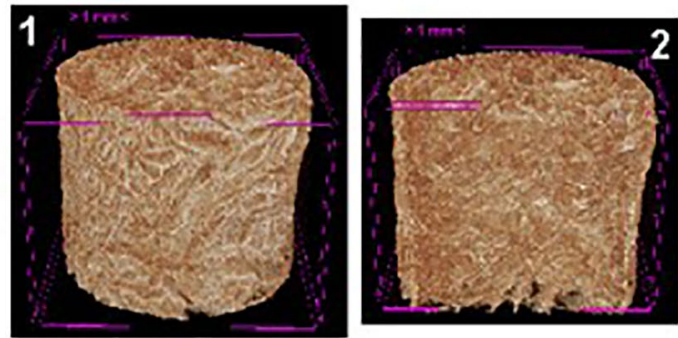

( $\mathrm{C} / \mathrm{N} / \mathrm{CNC} 2$ )

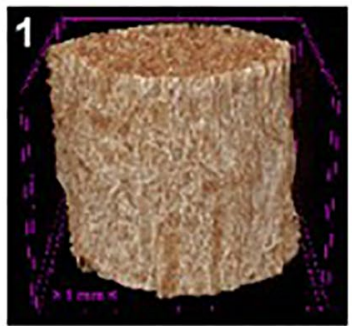

Porosity could also be determined, and it is shown in the graph from Figure 5. It can be noted that the values are all higher than $80 \%$, and the standard deviations are small, meaning that the pores were homogeneously distributed in the samples. Despite it seems that the values are all very

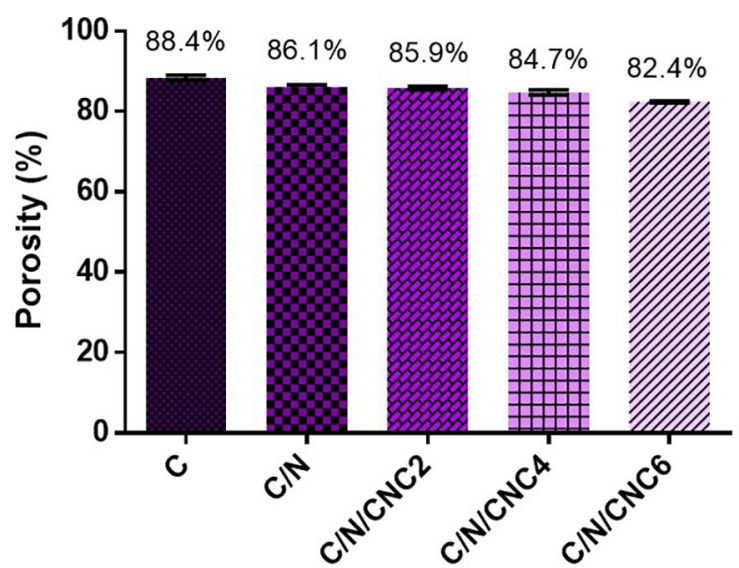

Figure 5. Porosity values of the hydrogels obtained by X-Ray microtomography. Results are given as mean $\pm \mathrm{SD}(\mathrm{n}=5)$.
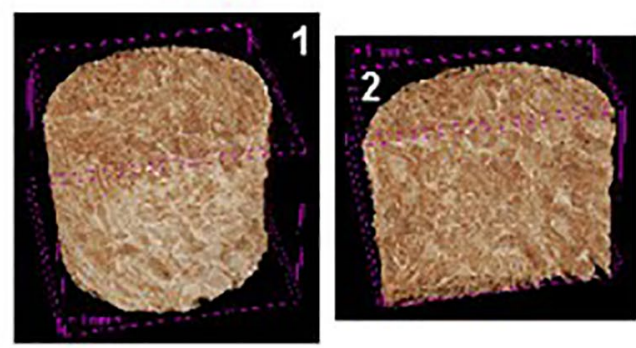

$(\mathrm{C} \mathbb{N})$
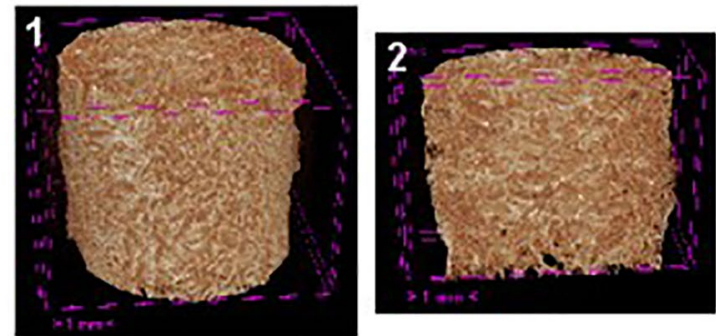

$(\mathrm{C} / \mathrm{N} / \mathrm{CNC} 4)$

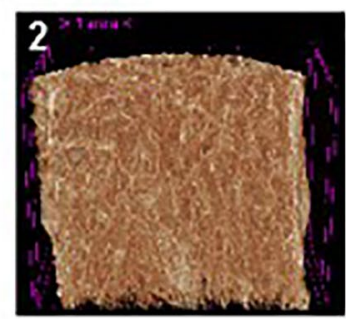

( $\mathrm{C} / \mathrm{N} / \mathrm{CNC} 6$ )

Figure 4. $3 \mathrm{D}$ view of $\mathrm{C}, \mathrm{C} / \mathrm{N}, \mathrm{C} / \mathrm{N} / \mathrm{CNC} 2, \mathrm{C} / \mathrm{N} / \mathrm{CNC} 4$ and $\mathrm{C} / \mathrm{N} / \mathrm{CNC} 6$ hydrogels. Front view (1) and cross section (2). 
close from each other (mean standard deviation $0.5 \%$ ), statistical differences were found between samples $(\mathrm{P}<0.05$ was considered significant). The addition of $2 \% \mathrm{CNC}$ did not change significantly the porosity of the hydrogel, compared to the sample without $\mathrm{CNC}$, but, except this case, there are statistical differences between all samples. The porosity was reduced with the addition of zinc nitrate and CNC.

As discussed on rheological results, the addition of zinc nitrate increased the viscosity of hydrogel and the crosslinking degree, what resulted in lower porosity. However, the CNC addition decreased the crosslinking degree as verified by the decreasing of hydrogels viscosity, but also reduced porosity, what was not expected.

Zeng et al. ${ }^{33}$ produced crosslinked chitosan membranes, using glutaraldehyde as the crosslinking agent. Authors changed glutaraldehyde concentration and evaluated porosity of the obtained membranes. They reported that increasing the crosslinking agent concentration, and, consequently the crosslinking degree, led to a reduction in the porosity. This behavior was similar to the observed with hydrogels in the present work and was attributed to the formation of a continuous and more compact network, resulting in a less porous structure.

\subsubsection{Scanning Electron Microscopy (SEM)}

The scanning electron micrographs of the hydrogels' fracture surfaces are shown in Figure 6. As already observed on microtomography images, the images of different hydrogels did not show any change in terms of size, quantity or distribution of the pores, so, it can be concluded that zinc nitrate and $\mathrm{CNC}$ addition did not change the chitosan hydrogels morphology. Similar morphology was observed in the study of Shen et al. ${ }^{34}$, which produced chitosan-gelatin hydrogels via in situ precipitation. Bi et al. ${ }^{35}$ produced chitosan/collagen scaffolds for cartilage tissue engineering crosslinked with genipin and observed similar morphology, including pore wall with paper-like morphology.

\subsubsection{Swelling Test}

The average values and standard deviation of hydrogels swelling degree are in Figure 7. Comparing values of $\mathrm{C}$ and $\mathrm{C} / \mathrm{N}$ samples, it is observed that the zinc nitrate addition decreased about $41 \%$ the swelling degree $(\mathrm{p}<0.05)$. This result is in accordance with the rheological analyses (figures 2 and 3), which showed that the addition of zinc nitrate increased the crosslinking degree of chitosan hydrogels. It may also be related to the reduction in porosity. However, there were no statistical differences between CNC samples $(2,4$ and $6 \mathrm{wt} \%)$ and the swelling degree remained around $63 \%$ for these samples. The increase of crosslinking degree decreases the water absorption capacity due to the reduction of free $\mathrm{OH}$ groups present in chitosan and $\mathrm{CNC}$, and the formation of a more rigid structure.
The addition of CNC caused a decrease in the crosslinking degree, verified by the viscosity decrease for hydrogels with CNC. Decreasing crosslinking degree would increase the swelling degree. However, increasing CNC concentration would increase the stiffness on hydrogel walls, which would decrease swelling ability. Thus, due to the coexistence of conflicting effects, there was no significant difference in the swelling degree, as expected.

\subsubsection{Uniaxial Compression Test}

The average values and standard deviation of hydrogels maximum strain and stress are shown in Figure 8.

The average values for maximum strain and stress were $27.4 \%$ and $28.0 \mathrm{kPa}$, respectively. Statistical analysis did not show significant differences between the samples. It is concluded that zinc nitrate and $\mathrm{CNC}$ addition did not influence the mechanical properties of the chitosan hydrogels. As observed by microtomography, the porosity suffered minor changes with the introduction of zinc nitrate and $\mathrm{CNC}$, meaning that the general structure of the hydrogels was not changed. The maintenance of general structure could also be observed in SEM images. For this reason, mechanical properties were expected not to suffer great changes; so the values of maximum strain and stress showed above are in accordance to the previous results reported.

Yue et al. ${ }^{36}$ reported significant improve on compression stress for sodium alginate-polyvinyl alcohol hydrogels reinforced with cellulose nanofibers (CNF). The enhancement in mechanical compression property was attributed to well dispersed CNF into the hydrogel structure.

Herein, what could have happened is an insufficient dispersion of CNC into the matrix, due to the high CNC concentration used. We may assume that $\mathrm{CNC}$ did not introduce imperfections in the hydrogel structure; however, it was not sufficiently well dispersed to improve mechanical properties.

The absence of improvements in mechanical properties due to inefficient filler dispersion was also reported by Jiang et al. ${ }^{37}$. When CNC is not satisfactorily dispersed, the material obtained behaves like composites reinforced with micronsized fillers, and no significant differences are observed in the final mechanical property of the material.

\subsubsection{In Vitro Degradation}

The results of hydrogels degradation are shown in Figure 9, which shows the average values and standard deviation of remaining mass (\%) as a function of time.

It can be observed in the curves from Figure 7 that the zinc nitrate and $\mathrm{CNC}$ addition did not influence the chitosan hydrogels degradation $(\mathrm{P}>0.05)$. All hydrogels degradation curves had similar behavior and after 56 days of testing, the mass remained at around $22 \%$ of the initial mass. An accelerated mass loss was observed in the first 30 days. 

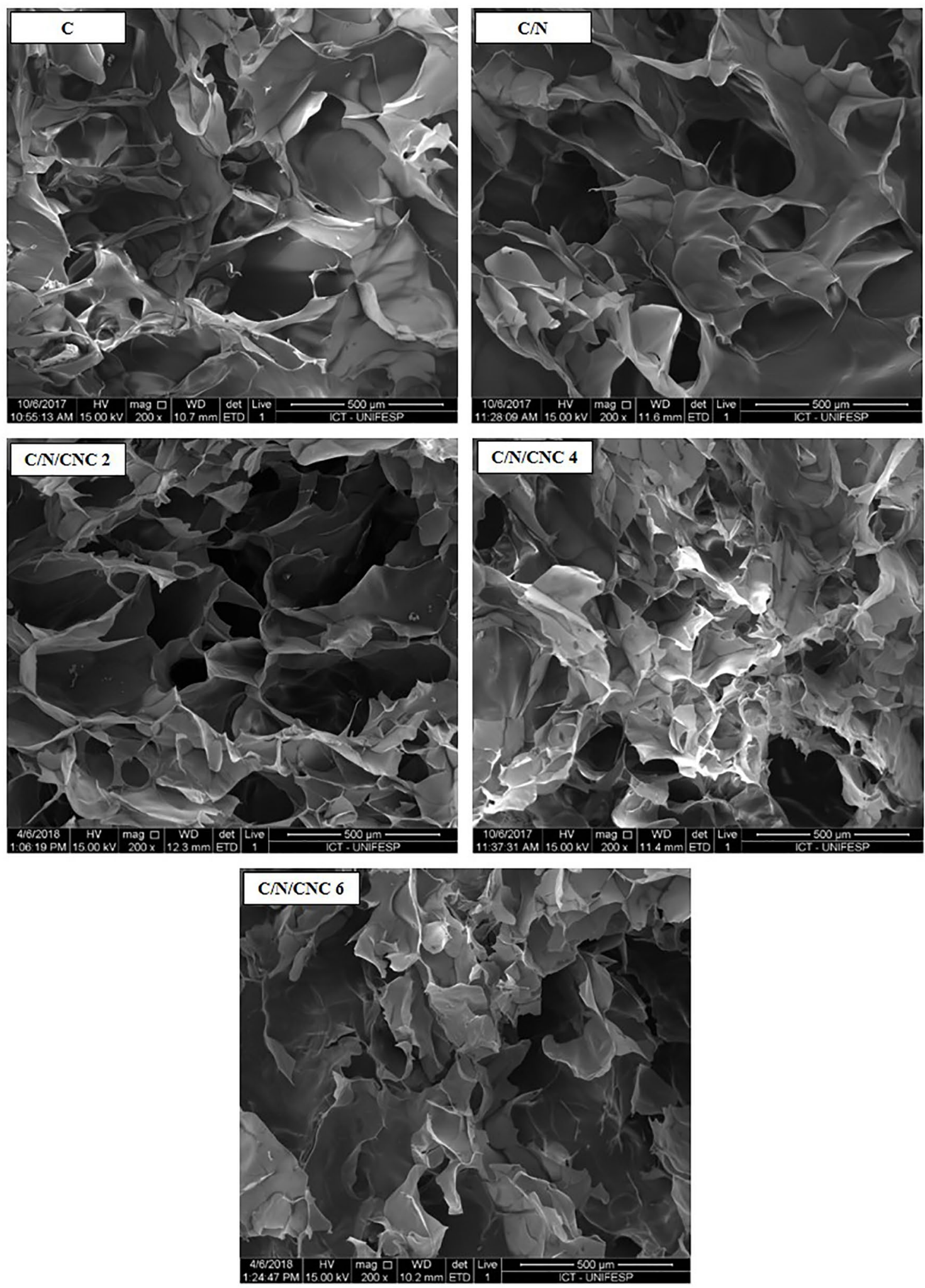

Figure 6. Fracture surface micrographs of chitosan hydrogels. Magnification of 200x. 


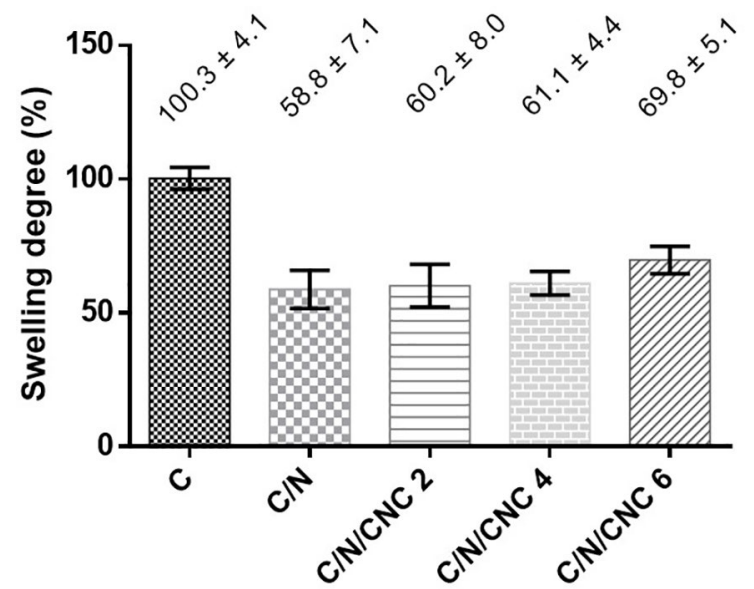

Figure 7. Swelling degree of hydrogels.
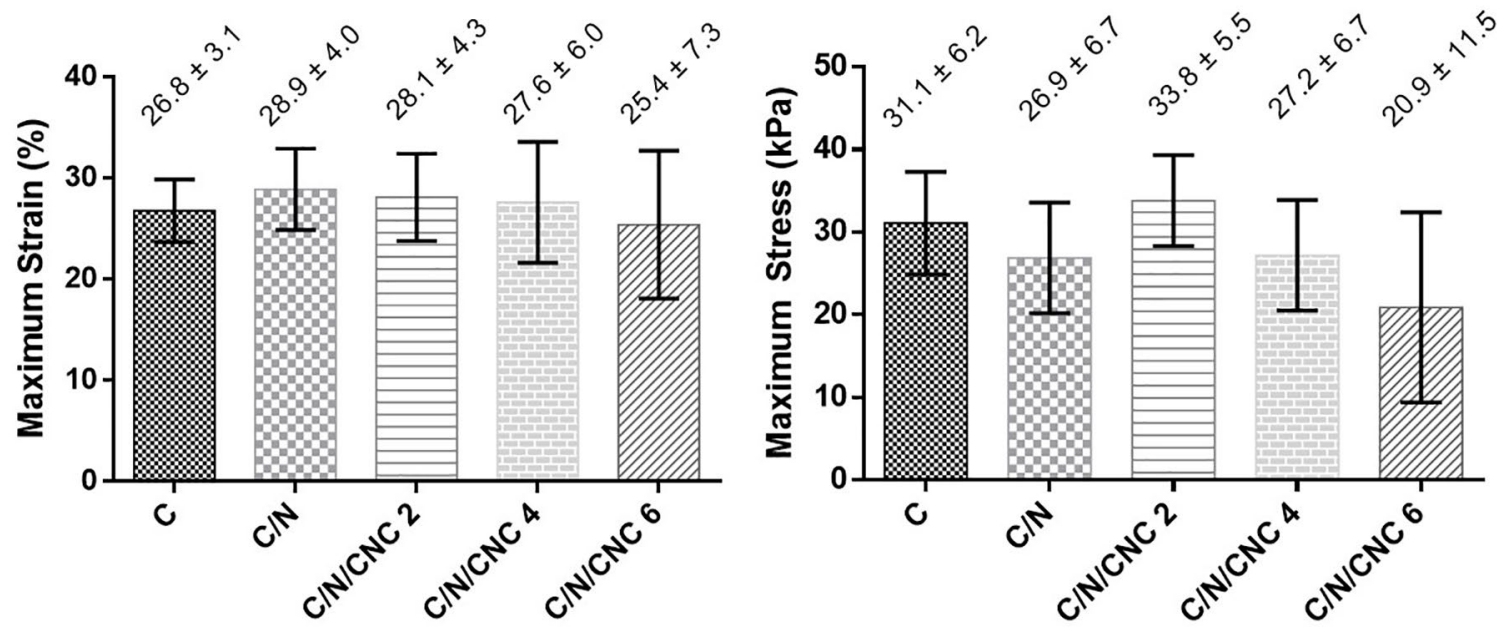

Figure 8. Maximum strain (\%) and maximum stress (\%) of hydrogels.

The behavior of the degradation curves can be described by Equation 3. This equation was taken from the curve of hydrogel $\mathrm{C}$, however, as there is no significant differences between the degradation curves, that equation describes all curves behavior.

$$
Y=\frac{100}{1+\left(10^{(1,4359+0,0714 X)}\right)}
$$

Since it was already demonstrated that chitosan degrade in vivo in a period of 6 months $^{38,39}$, it is possible to extrapolate the equation taken from de degradation of hydrogel $\mathrm{C}$ and find the value of $5 \times 10^{-13} \%$ mass for 180 days for the in vitro studies. This result allows supposing that chitosan hydrogels will properly degrade in vivo within 180 days.

\section{Conclusion}

Chitosan hydrogels were crosslinked with glutaraldehyde, and zinc nitrate hexahydrate was used as a catalyst in the

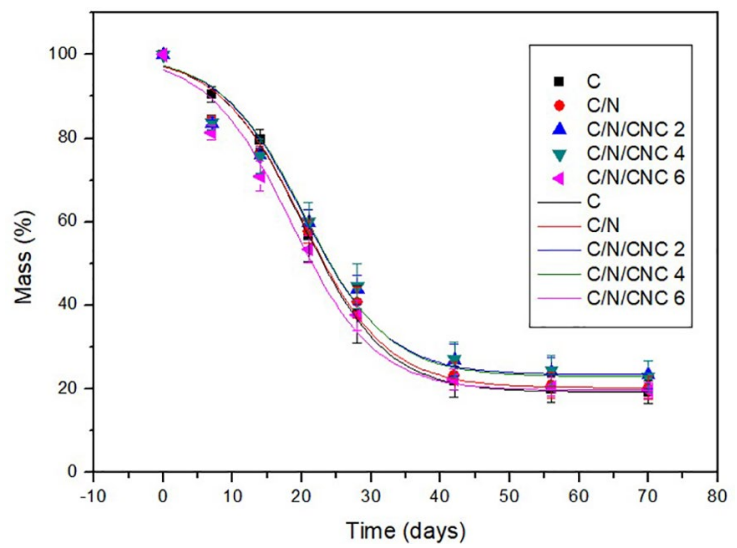

Figure 9. In vitro degradation curve of hydrogels.

crosslinking reaction. $\mathrm{CNC}$ was chosen as nanofillers. Rheology analysis showed that the addition of zinc nitrate increases viscosity and crosslinking degree of chitosan hydrogel, while $\mathrm{CNC}$ addition oppositely affects both properties. However, in both cases, the porosity is reduced. Meanwhile, the morphology, evaluated by SEM and microtomography, did not suffer significant changes for all evaluated conditions, showing uniform construction. Due to the coexistence of conflicting effects, swelling degree was not changed after the introduction of $\mathrm{CNC}$; however, this value was reduced about $41 \%$ after the addition of zinc nitrate to neat chitosan. Compressive mechanical properties remained constant, presumably because of an inefficient dispersion of $\mathrm{CNC}$ within the samples. As already expected, being a result of similar morphology, in vitro degradation test showed that all hydrogels degradation curves had similar behavior. Thus, the presence of zinc nitrate increased crosslinking degree proving that it is not only a catalyst but also participates in the crosslinking reaction of chitosan hydrogels. Accordingly, 
using zinc nitrate, it may be possible to achieve satisfactory crosslinking degrees with reduced amounts of glutaraldehyde, which has high toxicity.

\section{Acknowledgments}

We would like to thank the Nanostructured Soft Materials Laboratory, Brazilian Nanotechnology National Laboratory, LNNano, for the use of X-Ray Microtomography facility.

\section{References}

1. Nascimento MHM, Lombello CB. Hidrogéis a base de ácido hialurônico e quitosana para engenharia de tecido cartilaginoso. Polimeros. 2016;26(4):360-370.

2. Liu M, Zeng X, Ma C, Yi H, Ali Z, Mou X, et al. Injectable hydrogels for cartilage and bone tissue engineering. Bone Research. 2017;5:17014.

3. Elieh-Ali-Komi D, Hamblin MR. Chitin and Chitosan: Production and Application of Versatile Biomedical Nanomaterials. International Journal of Advanced Research (Indore). 2016;4(3):411-427.

4. Patrulea V, Ostafe V, Borchard G, Jordan O. Chitosan as a starting material for wound healing applications. European Journal of Pharmaceutics and Biopharmaceutics. 2015;97(Pt B):417-426.

5. Yang XL, Ju XJ, Mu XT, Wang W, Xie R, Liu Z, et al. CoreShell Chitosan Microcapsules for Programmed Sequential Drug Release. ACS Applied Materials \& Interfaces. 2016;8(16):1052410534.

6. Baldino L, Concilio S, Cardea S, De Marco I, Reverchon E. Complete glutaraldehyde elimination during chitosan hydrogel drying by $\mathrm{SC}-\mathrm{CO}_{2}$ processing. The Journal of Supercritical Fluids. 2015;103:70-76.

7. Mirzaei EB, Ramazani ASA, Shafiee M, Danaei M. Studies on Glutaraldehyde Crosslinked Chitosan Hydrogel Properties for Drug Delivery Systems. International Journal of Polymeric Materials and Polymeric Biomaterials. 2013;62(11):605-611.

8. Xu GG, Yang CQ, Deng Y. Applications of bifunctional aldehydes to improve paper wet strength. Journal of Applied Polymer Science. 2002;83(12):2539-2547.

9. Qiu YF, Qin YY, Ma Z, Xia WJ. Chitosan-supported Zinc Nitrate: Preparation and Catalyst for Condensation Reaction of Aldehydes, Amines, and Terminal Alkynes Leading to the Formation of Propargylamines. Chemistry Letters. 2014;43(8):1284-1286.

10. Maryam M, Suriani AB, Zobir SAM, Mahmood MR. Synthesis of Carbon Nanotubes Using Zinc Nitrate as a Catalyst by Aerosol-Assisted Catalytic Single Furnace CVD. Advanced Materials Research. 2012;576:349-352.

11. Reinoso DM, Damiani DE, Tonetto GM. Synthesis of biodiesel from soybean oil using zinc layered hydroxide salts as heterogeneous catalysts. Catalysis Science \& Technology. 2014;4(6):1803-1812.

12. Celebi H, Kurt A. Effects of processing on the properties of chitosan/cellulose nanocrystal films. Carbohydrate Polymers. 2015;133:284-293.
13. Wang W, Jung J, Zhao Y. Chitosan-cellulose nanocrystal microencapsulation to improve encapsulation efficiency and stability of entrapped fruit anthocyanins. Carbohydrate Polymers. 2017;157:1246-1253.

14. Habibi Y, Lucia LA, Rojas OJ. Cellulose Nanocrystals: Chemistry, Self-Assembly, and Applications. Chemical Reviews. 2010;110(6):3479-3500.

15. Moon RJ, Martini A, Nairn J, Simonsenf J , Youngblood J. Cellulose nanomaterials review: structure, properties and nanocomposites. Chemical Society Reviews. 2011;40(7):39413994.

16. Durán N, Lemes AP, Seabra AB. Review of cellulose nanocrystals patents: preparation, composites and general applications. Recent Patents on Nanotechnology. 2012;6(1):16-28.

17. Grishkewich N, Mohammed N, Tang J, Tam KC. Recent advances in the application of cellulose nanocrystals. Current Opinion in Colloid \& Interface Science. 2017;29:32-45.

18. Geng CZ, Hu X, Yang GH, Zhang Q, Chen F, Fu Q. Mechanically reinforced chitosan/cellulose nanocrystals composites with good transparency and biocompatibility. Chinese Journal of Polymer Science. 2015;33(1):61-69.

19. Fernandes SCM, Freire CSR, Silvestre AJD, Pascoal Neto C, Gandini A, Berglund LA, et al. Transparent chitosan films reinforced with a high content of nanofibrillated cellulose. Carbohydrate Polymers. 2010;81(2):394-401.

20. Li Q, Zhou J, Zhang L. Structure and properties of the nanocomposite films of chitosan reinforced with cellulose whiskers. Journal of Polymer Science Part B: Polymer Physics. 2009;47(11):1069-1077.

21. Kargarzadeh H, Sheltami RM, Ahmad I, Abdullah I, Dufresne A. Cellulose nanocrystal: A promising toughening agent for unsaturated polyester nanocomposite. Polymer. 2015;56:346357.

22. Anžlovar A, Huskić M, Žagar E. Modification of nanocrystalline cellulose for application as a reinforcing nanofiller in PMMA composites. Cellulose. 2016;23(1):505-518.

23. George J, Sabapathi SN. Cellulose nanocrystals: synthesis, functional properties, and applications. Nanotechnology, Science and Applications. 2015;8:45-54.

24. Durán N, Lemes AP, Durán M, Freer J, Baeza J. A minireview of cellulose nanocrystals and its potential integration. Journal of the Chilean Chemical Society. 2011;56(2):672-677.

25. Ridolfi DM, Lemes AP, de Oliveira S, Justo GZ, Palladino MV, Durán N. Electrospun poly(ethylene oxide)/chitosan nanofibers with cellulose nanocrystals as support for cell culture of 3T3 fibroblasts. Cellulose. 2017;28(8):3353-3365.

26. Asma C, Meriem E, Mahmoud B, Djaafer B. Physicochemical characterization of gelatin-CMC composite edibles films from polyion-complex hydrogels. Journal of the Chilean Chemical Society. 2014;59(1):2279-2283.

27. Mirhosseini H, Tan CP, Hamid NSA, Yusof S. Effect of Arabic gum, xanthan gum and orange oil contents on ?-potential, conductivity, stability, size index and $\mathrm{pH}$ of orange beverage emulsion. Colloids and Surfaces A: Physicochemical and Engineering Aspects. 2008;315(1-3):47-56. 
28. Montanheiro TLA, Montagna LS, de Farias MA, Magalhães JA, Tada DB, Passador FR, et al. Cytotoxicity and physico-chemical evaluation of acetylated and pegylated cellulose nanocrystals. Journal of Nanoparticle Research. 2018;20:206.

29. Grillet AM, Wyatt NB, Gloe LM. Polymer Gel Rheology and Adhesion. In: De Vicente J, ed. Rheology. London: InTech; 2012. p. 59-80.

30. Zhou C, Wu Q, Yue Y, Zhang Q. Application of rod-shaped cellulose nanocrystals in polyacrylamide hydrogels. Journal of Colloid and Interface Science. 2011;353(1):116-123.

31. Zhou C, Wu Q, Zhang Q. Dynamic rheology studies of in situ polymerization process of polyacrylamide-cellulose nanocrystal composite hydrogels. Colloid and Polymer Science. 2011;289(3):247-255.

32. Stock SR. Recent advances in X-ray microtomography applied to materials. International Materials Reviews. 2008;53(3):129-181.

33. Zeng M, Fang Z. Preparation of sub-micrometer porous membrane from chitosan/polyethylene glycol semi-IPN. Journal of Membrane Science. 2004;245(1-2):95-102.

34. Shen ZS, Cui X, Hou RX, Li Q, Deng HX, Fu J. Tough biodegradable chitosan-gelatin hydrogels via in situ precipitation for potential cartilage tissue engineering. RSC Advances. 2015;5(69):55640-55647.
35. Bi L, Cao Z, Hu Y, Song Y, Yu L, Yang B, et al. Effects of different cross-linking conditions on the properties of genipincross-linked chitosan/collagen scaffolds for cartilage tissue engineering. Journal of Materials Science: Materials in Medicine. 2011;22(1):51-62.

36. Yue Y, Han J, Han G, French AD, Qi Y, Wu Q. Cellulose nanofibers reinforced sodium alginate-polyvinyl alcohol hydrogels: Core-shell structure formation and property characterization. Carbohydrate Polymers. 2016;147:155-164.

37. Jiang L, Morelius E, Zhang J, Wolcott M, Holbery J. Study of the Poly(3-hydroxybutyrate-co-3-hydroxyvalerate)/Cellulose Nanowhisker Composites Prepared by Solution Casting and Melt Processing. Journal of Composite Materials. 2008;42(24):26292645.

38. Durkut S, Elçin YM, Elçin AE. Biodegradation of chitosantripolyphosphate beads: in vitro and in vivo studies. Artificial Cells, Blood Substitutes, and Immobilization Biotechnology. 2006;34(2):263-276.

39. Malafaya PB, Reis RL. Bilayered chitosan-based scaffolds for osteochondral tissue engineering: Influence of hydroxyapatite on in vitro cytotoxicity and dynamic bioactivity studies in a specific double-chamber bioreactor. Acta Biomaterialia. 2009;5(2):644-660. 